\title{
LUNAR SURFACE SAMPLING FEASIBILITY EVALUATION METHOD FOR Chang'E-5 MISSION
}

\author{
J. Wang ${ }^{1, *}$, C. Ma ${ }^{1}$, Z. Zhang ${ }^{1}$, Y. Wang ${ }^{2}$, M. Peng ${ }^{2}$, W. Wan ${ }^{2}$, X. Feng ${ }^{1}$, X. Wang ${ }^{1}$, X. He ${ }^{1}$, Y. You ${ }^{1}$ \\ ${ }^{1}$ Beijing Aerospace Control Center, Beijing, China - (15210106156@139.com, chuanling_ma@139.com, zining_zhang@139.com, \\ 130123_feng@163.com, xiaoxue_wang@139.com, heximing15@nudt.edu.cn, ywyykathy@yeah.net) \\ ${ }^{2}$ State Key Laboratory of Remote Sensing Science, Institute of Remote Sensing and Digital Earth, Chinese Academy of Sciences, \\ Beijing, China - (wangyx716, pengman, wanwh)@ radi.ac.cn
}

\section{Commission III, ICWG III/II}

KEY WORDS: Chang'E-5 Mission, Lunar Surface Sampling, Sampling Feasibility, Digital Elevation Model, manipulator arm

\begin{abstract}
:
As China's first unmanned spacecraft to collect lunar surface samples and return them to Earth, the Chang'E-5 detector is a crucial probe that will complete lunar surface sampling in China's lunar exploration project. This lunar sampling will be the first successful lunar surface sampling return mission in China. Sampling decisions needs to be made based on topographical analysis results and characteristics of the area to be explored. Due to the unknown extraterrestrial terrain and uncertainty of sampled objects, we propose a sampling feasibility estimation for safely implementing lunar surface sampling.

Our strategy took into account the influence of factors that may interfere with the sampling process, and provided quantitative assessment of the sampling feasibility for the area to be explored. We combined the three-dimensional topography of the lunar surface with five parameters of the sampling area, flatness, slope, slope aspect, accessibility of the mechanical arm distal end, and safety of sampling conditions. The first three values were calculated based on a digital elevation model (DEM) of the landing area generated using stereo images. The other values were computed based on the mechanical properties of the arm and kinematic analysis of its articulated joints. Based on the above-mentioned quantitative parameters, they were weighed to obtain an evaluation value for the sampling feasibility of each DEM pixel. Meanwhile, a multichannel sampling area analysis graph was generated that combined all the above indicators as well as the sampling feasibility values, which provides a visualization for determining detection targets in the Chang'E-5 sampling mission.
\end{abstract}

\section{INTRODUCTION}

In order to explore the distribution of space resources and understand the evolutionary history of the universe, human beings have performed sampling and analysis of soil and rock samples from the lunar surface on several occasions. It is important in deep space exploration to obtain and analyze samples using sampling mechanisms. International space organizations and research institutes have invested considerable energy in deep space exploration sampling and return technology, and have achieved many valuable results. From 1969 to 1972, the United States brought back $382 \mathrm{~kg}$ (842 pounds) of lunar soil samples from the lunar surface through six Apollo missions. In addition, three automated spacecrafts from the former Soviet Union brought back important samples from three different locations on the moon, totaling to $300 \mathrm{~g}$ (about $3 / 4$ pounds). (Allton $\mathrm{J} \mathrm{H}$ and Bevill $\mathrm{T} \mathrm{J}$, 2003; Bugos G, et al, 2013). In 2014, the Rosetta probe landed on the surface of Comet 67P to perform in-situ soil analyses. (Yoseph B C, et al, 2009). Human deep space exploration has basically covered a vast majority of planets, satellites, and asteroids in our solar system.

Since the lunar exploration project was officially launched in 2004, China has completed the lunar "around" and "falling" exploration missions successfully and collected a large number of new data on lunar surface topography and landforms. (Dai $S$ W, et al, 2014). In 2009, while performing the second phase of lunar exploration, China formally launched pre-research for the third phase of its lunar exploration program. It is expected that by the end of 2019, China will launch its first spacecraft with the intent of a return from the lunar surface following sampling. (Yu Dengyun, et.al, 2007; Peijian and Peng Jing, 2006).

Based on the lunar exploration project in China, this paper focuses on the key issues in lunar surface sampling: the method of evaluating the admissibility of sampling area, and using a digital elevation model (DEM) image for sampling area to generate an feasibility image of the sampling area. By interpreting the feasibility image, assisting the teleoperator to select the follow-up sampling points and plan the motion of the manipulator arm, the implementation efficiency of sampling area analysis is improved.

\section{SAMPLING AREA ASSESSMENT METHOD}

The configuration of the Chang'E-5 detector system at launch is shown in Figure 1. The top-down configuration consists of an ascender, a lander, a returner, and an orbiter. Chang'E-5 will be launched from the Hainan launch site by a new generation of Long March-5 launch vehicle. The whole flight process has gone through 11 orbital changing stages and the total flight

\footnotetext{
* Corresponding author.
} 
time will be approximately one month. The probe is divided into an orbiter and return assembly and a lander and lifter assembly. The orbiter-return assembly will continue to fly in a $200 \mathrm{~km}$ circular orbit, while the landing-lifter assembly will decelerate into an elliptical orbit of $15 \times 200 \mathrm{~km}$ in time, and allow the aircraft to implement power descent and soft landing on the lunar surface. After landing, the landing ascent assembly will carry out scientific exploration, complete the collection and packaging of lunar samples, and prepare for lunar takeoff. (Cai Ting-ni, et al, 2018).

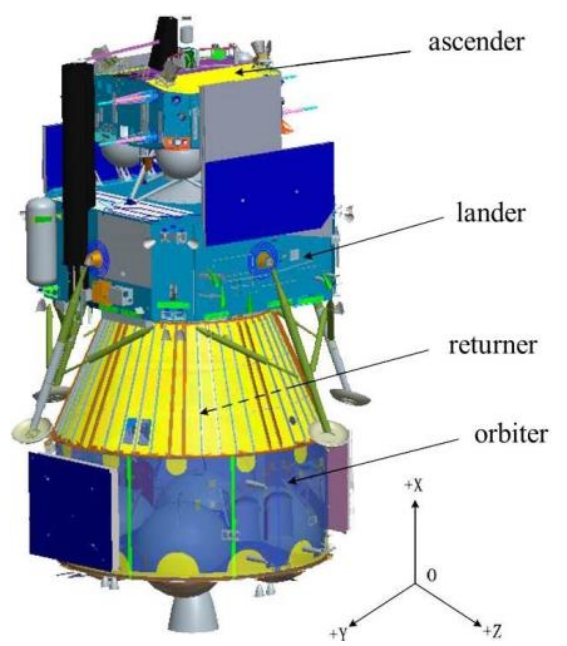

Figure 1. Emission configuration of Chang'E-5 detector

The Chang'E-5 lunar sampling package includes two processes: drilling sampling package and table sampling package. The lunar sampling and packaging work will involve the collection of lunar samples with specific weights, which should be completed in 2 days. Because it is time-constrained, it will be difficult to execute, owing to many uncertainties and a complex collaborative relationship. Combined with the characteristics of the lunar sampling process, parameters such as the threedimensional terrain, flatness of the sampling area, slope of the sampling point, gradient and gray level, accessibility by the end of the manipulator, and safety of the sampling conditions will be employed to synthetically generate multichannel images and comprehensively evaluate the recoverability of the sampling area. A flow chart depicting the feasibility of the sampling area analysis is shown in Figure 2.

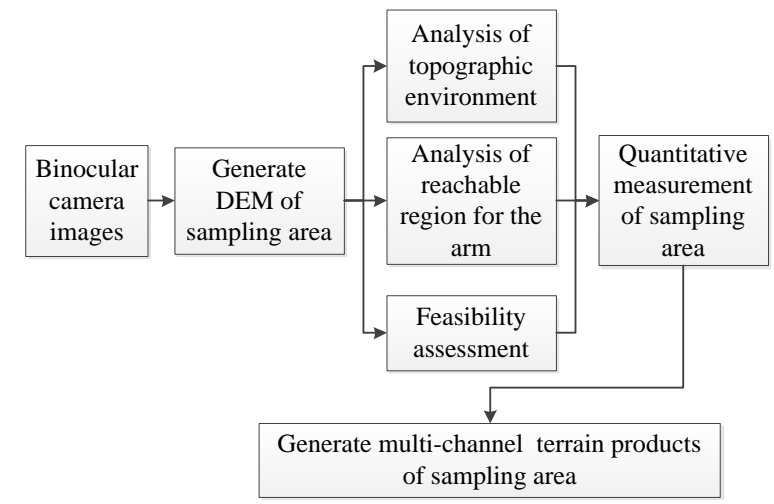

Figure 2. Sampling area feasibility analysis flow chart
Geomorphological analysis of the sampling area is mainly performed to analyze topographic fluctuations and steepness of the sampling area. It includes three calculations: flatness, slope, and slope direction calculations. Using the spatial coordinates corresponding to the pixels (grids) in the DEM images and given the size of the calculation window, all pixels in the window are fitted to the plane, the average distance between all the pixels in the window and the fitting plane is calculated, and the obtained value is considered as the flatness value corresponding to the central pixel of the window. Moreover, the angle between the fitting plane and the horizontal plane is considered as the slope value of the central pixel of the window, and the fitting level is obtained. The angle between the projection and the initial direction of the surface normal vector on the horizontal plane is defined as the slope direction of the central pixel, and the window traverses the whole sampling area to obtain the flatness, slope, and slope direction of the whole sampling area.

\subsection{Reachability analysis of the arm}

The reachability analysis of the arm is used to analyze the workspace and reachable range of the manipulator end-actuator. First, according to the structural characteristics of the arm, it is judged whether the space target point in the vertical direction ( $Z_{0}$ direction) and horizontal direction $\left(Z_{0}=p_{z}\right)$ belong to the working space of the arm. Then, according to the configuration constraints of the end-actuator parallel to the slope of the sampling point and the slope and direction information, the attitude angle of the end-actuator relative to the base coordinate system of the arm is calculated. The inverse kinematics method is used to determine the joint angle of each joint of the arm. According to the motion range of each joint angle, it can be determined if the target point exceeds the pose limit of the manipulator.

\subsection{Feasibility assessment}

The feasibility assessment of sampling points is used to determine if the lander body will interfere with the arm during sampling. First, using the joint angles of the arm, the coordinates of the connecting rod and the end-effector are obtained. Then, the linear equation for the connecting rod and the end-effector is obtained. According to the structural relationship between the base coordinate system and the lander, the plane equation of the lander is also obtained. Therefore, the intersection point of the connecting rod and the end-effector is obtained, and the position of the intersection point on the arm and the lander can be determined to complete the ontology interference detection.

\subsection{Quantitative measurement of sampling points}

If the selected sampling point does not exceed the position and attitude limit of the manipulator and does not collide with the manipulator and the lander during the sampling process, the flatness of the sampling point is first normalized. Then, according to formula (1), the feasibility parameters of the sampling points are calculated:

$$
W(x, y)=\alpha \bar{V}(x, y)+(1-\alpha) q(x, y)
$$

where $\quad(x, y)=$ image coordinates of the sampling point

\section{$(x, y)=$ image coordinates of the sampling point}

\subsection{Analysis of the topographic environment}




$$
\begin{aligned}
& \alpha=\text { weight coefficient } \\
& \bar{V}(x, y)=\text { normalized flatness } \\
& q(x, y)=\text { touchdown safety parameters }
\end{aligned}
$$

\section{ANALYSIS OF TERRAIN ENVIRONMENT}

\subsection{Flatness Calculation}

Flatness is used to reflect the distance dispersion relationship between the corresponding spatial coordinates of each pixel in the window area and the fitting plane of the window area. The larger the flatness value (i.e., the greater the dispersion of the distance between the corresponding spatial point and the plane of the pixel) the more uneven is the window area; conversely, the smaller the flatness value (i.e., the smaller the dispersion of the distance between the corresponding spatial point and the plane of the pixel) the flatter the window area. The process for flatness calculation is as follows:

Using DEM of the sampling area, the calculation window size is set to $w \times w$, and the least square method is used to fit all pixels in the calculation window. (Li Wei, et al, 2017). If $\mathrm{n}$ points to be fitted in the calculation window are $\left(x_{i}, y_{i}, z_{i}\right)$, $i=1,2,3, \ldots n$, then the fitting plane equation to be solved can be expressed as $a x+b y+c z+1=0$, which can be further written as:

$$
\left[\begin{array}{ccc}
x_{1} & y_{1} & z_{1} \\
\vdots & \vdots & \vdots \\
x_{n} & y_{n} & z_{n}
\end{array}\right]\left[\begin{array}{l}
a \\
b \\
c
\end{array}\right]=\left[\begin{array}{c}
-1 \\
-1 \\
-1
\end{array}\right]
$$

Formula (1) left multiplication $\left[\begin{array}{ccc}x_{1} & y_{1} & z_{1} \\ \vdots & \vdots & \vdots \\ x_{n} & y_{n} & z_{n}\end{array}\right]^{T}$ on both sides, we obtain:

$$
\left[\begin{array}{ccc}
x_{1} & \cdots & x_{n} \\
y_{1} & \cdots & y_{n} \\
z_{1} & \cdots & z_{n}
\end{array}\right]\left[\begin{array}{ccc}
x_{1} & y_{1} & z_{1} \\
\vdots & \vdots & \vdots \\
x_{n} & y_{n} & z_{n}
\end{array}\right]\left[\begin{array}{l}
a \\
b \\
c
\end{array}\right]=\left[\begin{array}{ccc}
x_{1} & \cdots & x_{n} \\
y_{1} & \cdots & y_{n} \\
z_{1} & \cdots & z_{n}
\end{array}\right]\left[\begin{array}{l}
-1 \\
-1 \\
-1
\end{array}\right]
$$

After sorting out, we obtain:

$$
\left[\begin{array}{ccc}
\sum x_{i} x_{i} & \sum x_{i} y_{i} & \sum x_{i} z_{i} \\
\sum x_{i} y_{i} & \sum y_{i} y_{i} & \sum y_{i} z_{i} \\
\sum x_{i} z_{i} & \sum y_{i} z_{i} & \sum z_{i} z_{i}
\end{array}\right]\left[\begin{array}{l}
a \\
b \\
c
\end{array}\right]=\left[\begin{array}{l}
-\sum x_{i} \\
-\sum y_{i} \\
-\sum z_{i}
\end{array}\right]
$$

From equation (4), the coefficients of the plane equation can be obtained as follows:

$$
\left[\begin{array}{l}
a \\
b \\
c
\end{array}\right]=\left[\begin{array}{ccc}
\sum x_{i} x_{i} & \sum x_{i} y_{i} & \sum x_{i} z_{i} \\
\sum x_{i} y_{i} & \sum y_{i} y_{i} & \sum y_{i} z_{i} \\
\sum x_{i} z_{i} & \sum y_{i} z_{i} & \sum z_{i} z_{i}
\end{array}\right]^{-1}\left[\begin{array}{l}
-\sum x_{i} \\
-\sum y_{i} \\
-\sum z_{i}
\end{array}\right]
$$

Flatness is defined as the average of the vertical distance from all the pixels in the window to the fitting plane, which can be expressed as formula (6):

$$
V(x, y)=\frac{1}{N} \sum_{i \in W} \frac{\left|a x_{i}+b y_{i}+c z_{i}+d\right|}{\sqrt{a^{2}+b^{2}+c^{2}}}
$$

where $\left(x_{i}, y_{i}, z_{i}\right)=$ spatial coordinates of each pixel in a window

$w=$ window size

$V(x, y)=$ flatness of the central pixel of the current window

$N=$ number of pixels in the window participating in the calculation

\subsection{Slope Calculation}

The slope of the sampling area is used to reflect the steepness of the surface. The smaller the slope value, the gentler the slope; on the other hand, the larger the slope value, the steeper the slope. Using the spatial coordinates corresponding to the pixels in DEM image data and the specified calculation window size, all the pixels in the window are used to fit the plane, and the angle between the fitting plane and the horizontal plane is used as the slope value of the central pixel of the window. The range of slope value was: $\left[0,90^{\circ}\right]$. Using the plane: $a x+b y+c z+1=0$ fitted in Section 3.1, the slope is defined as the complementary angle of the angle between the normal vector of the fitted plane and its projection vector on the horizontal plane; then, the slope can be expressed as:

$$
\beta=\arctan \frac{\sqrt{\left(a^{2}+b^{2}\right)}}{c}
$$

\subsection{Slope Aspect Calculation}

The slope direction of the sampling area is used to reflect the corresponding direction of the slope surface. Using the spatial coordinates corresponding to the pixels in DEM image and the specified calculation window size, all the pixels in the window are used to fit the plane, and the angle between the projection of the fitting plane normal vector on the horizontal plane and the initial direction is taken as the slope value of the central pixel. The range of gradient value was: $\left[0,360^{\circ}\right]$.

Starting from the East direction ( $+x$ axis) and measuring counter-clockwise, the slope direction of each spatial point can be expressed as:

$$
\gamma=\arctan \frac{b}{a}
$$

\section{REACHABLE INTERVAL ANALYSIS}

In this paper, a 4-DOF arm was considered as an example to analyze the kinematics of the arm and perform reachable interval modeling. The sampling manipulator consists of four rotary joints, namely, a lumbar joint, shoulder joint, elbow joint and wrist joint, and two connecting rods.

\subsection{Dynamics Analysis of 4-DOF Arm}

The working mechanism of the lunar soil surface sampling manipulator includes folding and unfolding, unfolding sampling, and sample transmission. Because there are obstacles such as rocks on the lunar surface, during sampling, the arm usually needs to move the end-effector to a place 
where there were no rocks for sampling, which requires that the end-effector can reach the pre-selected specific target location. At the same time, during the sampling process, the end-effector also needs to maintain a specific attitude angle to dig into the lunar soil. Finally, in the process of sampling and transferring, the end-effector should maintain a vertical attitude to avoid scattering the lunar soil.

The linkage coordinate system of a 4-DOF arm can be constructed according to the D-H method. (Denavit $\mathrm{J}$ and Hartenberg, 1955). The structure sketch and the linkage coordinate system of the manipulator is shown in Figure 3.

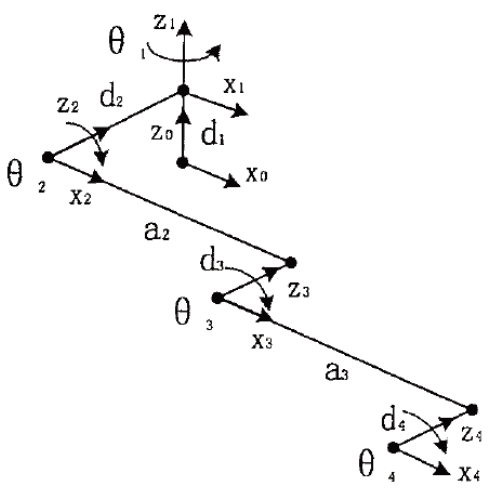

Figure 3. Structure sketch and connecting rod coordinate system of arm

The arm of the sampling manipulator was in the zero position (each joint angle was 0), and the arm axis was parallel to the $\mathrm{Z}$ axis and YOZ plane of the lander. Without considering the interference of other objects, the rotational range of each joint is as follows:

\begin{tabular}{|c|c|c|c|c|}
\hline & joint 1 & joint 2 & joint 3 & joint 4 \\
\hline $\begin{array}{c}\text { rotation } \\
\text { range }\end{array}$ & $-180-180$ & $-135-90$ & $-180-180$ & $-180-180$ \\
\hline
\end{tabular}

Table 1. Rotational range of each joint

\subsection{Calculation of the Arm Working Interval}

The two-dimensional coordinates of the arm in the operating surface $\left(O_{1} X_{1} Z_{1}\right)$ can be expressed as follows:

$$
\left[\begin{array}{c}
p_{x_{1}} \\
p_{z_{1}}
\end{array}\right]=\left[\begin{array}{c}
a_{2} c_{2}+a_{3} c_{2+3}+a_{4} c_{2+3+4} \\
-a_{2} s_{2}-a_{3} s_{2+3}-a_{4} s_{2+3+4}
\end{array}\right]
$$

By combining Formula 1 with the range of motion of joint 2, joint 3 and joint 4 , the contour coordinates of the reachable range in the operating plane can be obtained as formula 10 :

$$
\left[\begin{array}{l}
\bar{p}_{x_{1}} \\
\bar{p}_{z_{1}}
\end{array}\right]=\left\{\begin{array}{cc}
{\left[\begin{array}{c}
a_{2} \cos \left(-\frac{3}{4} \pi\right)+\left(a_{3}+a_{4}\right) \cos \left(-\frac{3}{4} \pi+\theta_{3}\right) \\
-a_{2} \sin \left(-\frac{3}{4} \pi\right)-\left(a_{3}+a_{4}\right) \sin \left(-\frac{3}{4} \pi+\theta_{3}\right)
\end{array}\right]} & \theta_{3} \in[-(\pi-\delta) 0] \\
{\left[\begin{array}{c}
\left(a_{2}+a_{3}+a_{4}\right) c_{2} \\
-\left(a_{2}+a_{3}+a_{4}\right) s_{2}
\end{array}\right]} & \theta_{2} \in\left[-\frac{3}{4} \pi \frac{1}{2} \pi\right] \\
{\left[\begin{array}{c}
a_{2} \cos \left(\frac{1}{2} \pi\right)+\left(a_{3}+a_{4}\right) \cos \left(\frac{1}{2} \pi+\theta_{3}\right) \\
-a_{2} \sin \left(\frac{1}{2} \pi\right)-\left(a_{3}+a_{4}\right) \sin \left(\frac{1}{2} \pi+\theta_{3}\right)
\end{array}\right]} & \theta_{3} \in[0 \pi-\delta]
\end{array}\right.
$$

The angle $\delta$ can be calculated using the following formula:

$$
\begin{gathered}
\delta=A \tan \left(\frac{2 a_{2} L \sin \left(\frac{3}{8} \pi\right)}{a_{2}^{2}+\left(a_{3}+a_{4}\right)^{2}-L^{2}}\right) \\
L=a_{2} \cos \left(\frac{3}{8} \pi\right)+\sqrt{a_{2}^{2} \cos ^{2}\left(\frac{3}{8} \pi\right)+\left(a_{3}+a_{4}\right)^{2}-a_{2}^{2}}
\end{gathered}
$$

According to the known DH parameters of the manipulator, the reachable range in the operating plane can be determined, as shown in Figure 4. The length of the position vector at the concave point in the graph is $\mathrm{L}$, and the amplitude angle of the position vector is $9 \pi / 8$.

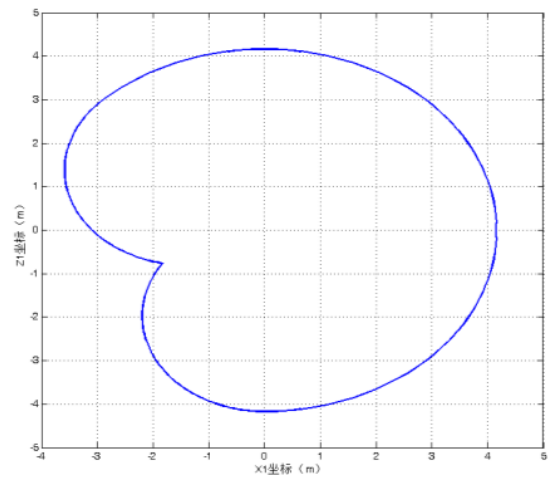

Figure 4. Reachable range in the operation plane

Based on the known DH parameters of the manipulator and the condition of the body parameters, the three-dimensional workspace in the base coordinate system can be determined, as shown in Figure 5.

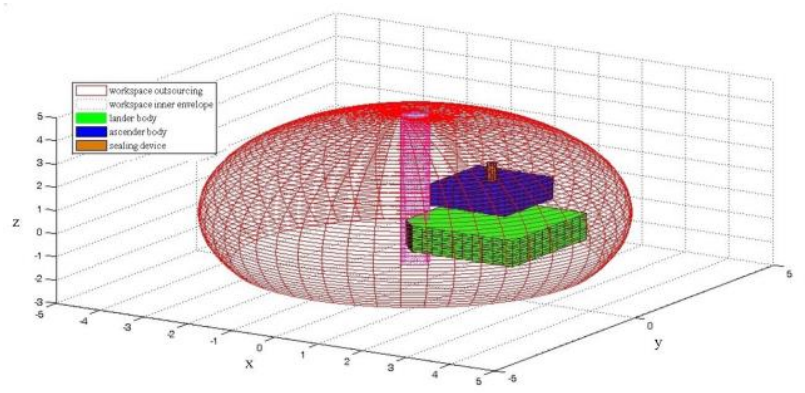

Figure 5. Three-dimensional workspace in the base coordinate system

The accessibility of spatial target points should be considered as an important parameter when making decisions regarding sampling locations. According to the workspace in the base coordinate system, the following steps can be performed to determine if the space points are reachable:

Step 1: Determine whether the coordinate components $p_{z}$ of the space target point $\left(p_{x}, p_{y}, p_{z}\right)$ satisfy the following inequalities:

$$
d_{1}-a_{2}-a_{3}-a_{4} \leq p_{z} \leq d_{1}+a_{2}+a_{3}+a_{4}
$$

If the inequality is satisfied, the coordinate components of the target point are located in the workspace of the manipulator, 
and the next step can be judged; otherwise, the target point cannot be reached.

Step 2: Determine whether the space target point $\left(p_{x}, p_{y}, p_{z}\right)$ is located in the workspace of the manipulator in the horizontal section $Z_{0}=p_{z}$ of the workspace, i.e., whether it satisfies the inequality:

$$
\begin{aligned}
& \left(d_{2}+d_{3}+d_{4}\right)^{2} \leq p_{x}{ }^{2}+p_{y}{ }^{2} \\
& \leq\left(d_{2}+d_{3}+d_{4}\right)^{2}+\left(a_{2}+a_{3}+a_{4}\right)^{2}-\left(p_{z}-d_{1}\right)^{2}
\end{aligned}
$$

If the inequality is satisfied, then the target point is reachable; otherwise, the target point is not reachable.

\section{FEASIBILITY ASSESSMENT}

Feasibility evaluation of the sampling target points comprises two main aspects. One is to determine whether the actual terrain of the projection area of the end-effector of the manipulator on the horizontal plane $O_{0} X_{0} Y_{0}$ of the lunar base coordinate system interferes with the sampling process of the manipulator. The other is to determine whether the lander's body will interfere with the manipulator during the sampling process.

\subsection{Intervention Test Based on Lunar Terrain}

On the premise that the spatial coordinates of the reachable target point are known, the lunar terrain of the sampling area can be judged based on the processing flow shown in Fig. 6 .

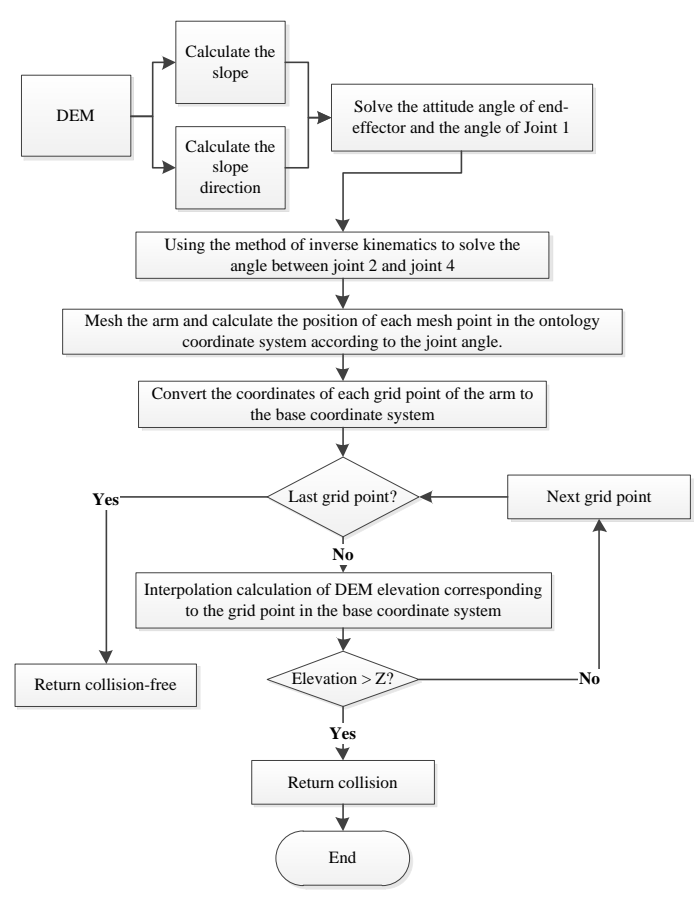

Figure 6. Flow chart of lunar interferometric detection

\subsection{Ontological Interference Test}

The arm may be interfered with by the lander body during the sampling process. It is necessary to detect the joint angle by inverse kinematics to determine whether there is an interference risk between the joint angle and the body. The process for ontology interference detection is shown in Figure 7.

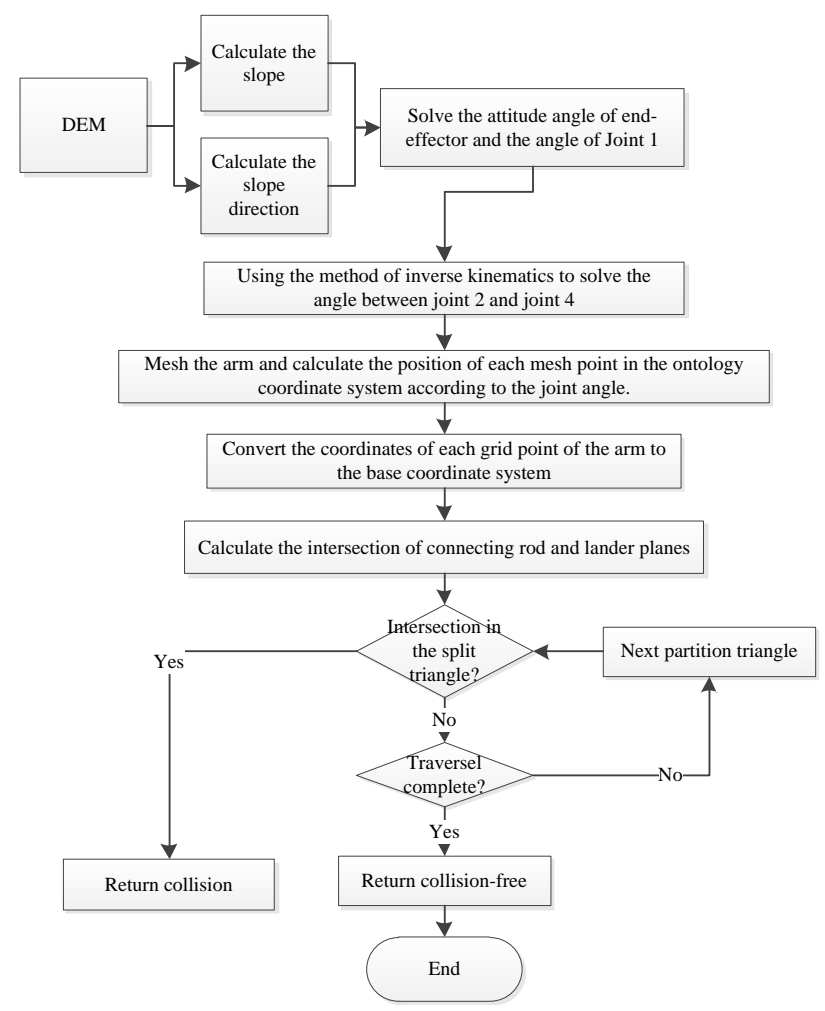

Figure 7. Diagram of the ontology interference detection process

\subsection{Calculation of the Safety Parameters of Touching Ground}

We set $\delta=\min \left(\delta_{L 3}, \delta_{2}\right) ; \delta$ uses the smaller value of the minimum distance between the connecting rod and the lander and the minimum distance between the end-effector and the ground. When the earth-touching sensor touches the ground, the distance between the center of the earth-touching sensor and the ground is radius $R_{D}$; then, the safety parameters of the manipulator touching the ground are defined as:

$$
q(x, y)=\frac{\delta}{R_{D}}
$$

When the parameter $q(x, y)$ is between 0 and 1. $q(x, y)=0$, which means that the connecting rod on the manipulator collides with the lander or the end-effector touches the ground and could not be sampled. With an increase in value, the safety of touching the ground increased.

\section{ANALYSIS OF SIMULATION RESULTS}

The flatness, accessibility and safety of the spatial coordinates were then calculated using the spatial coordinates corresponding to the pixels (grids) in the DEM image of the sampling area. The output was interpreted and processed, and the whole sampling area was traversed to generate an image representing the admissibility of the sampling area. The 
process of image generation for admissibility determination is shown in Figure 8.

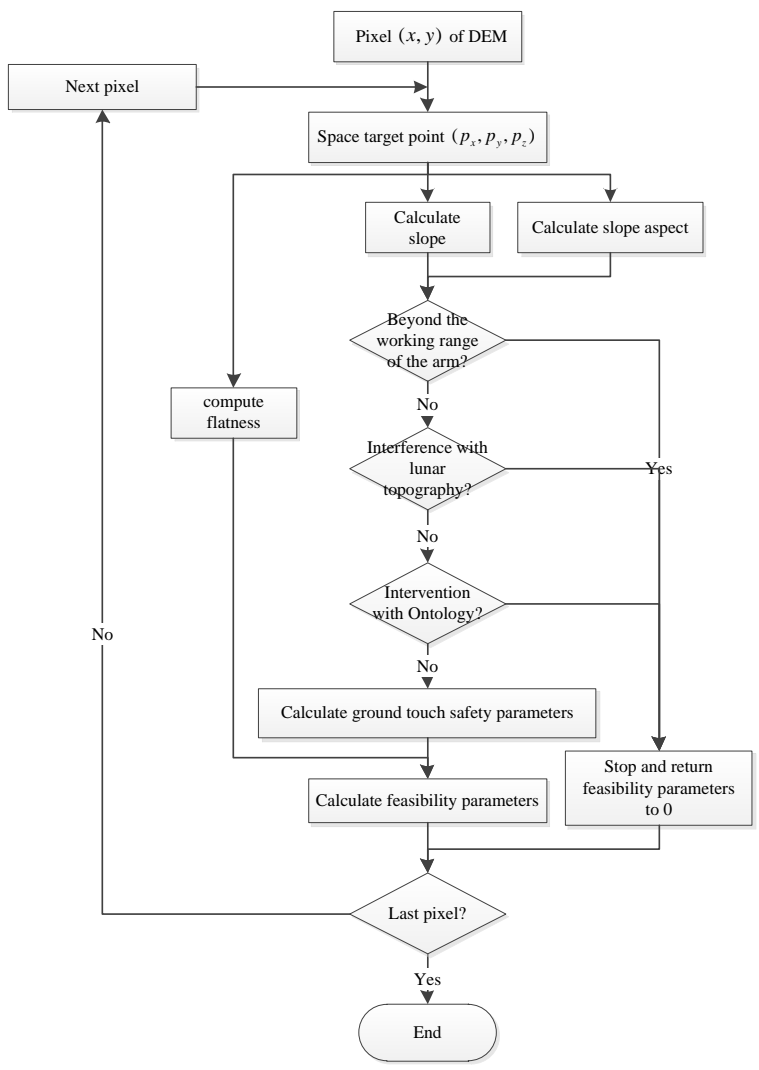

Figure 8. Flow chart for calculating topographic feasibility

Using the simulated lunar sampling DEM image (as shown in Figure 9), the admissibility image generated by the method presented in this paper is shown in Figure 10. The gray value of the recoverable image is determined by the flatness and accessibility of the sampling area and the safety of the manipulator. The safety channel is determined by the slope, aspect, and configuration of the manipulator. According to the principle that stones larger than $4 \mathrm{~cm}$ can not be recovered, the image sets its corresponding admissibility value in an interval, and displays the original admissibility image in the interval of the set admissibility value. Blue represents the exploitable area and red represents the less exploitable area. The missing part in the lower left corner of the admissibility graph is the unreachable and unsafe sampling interval.

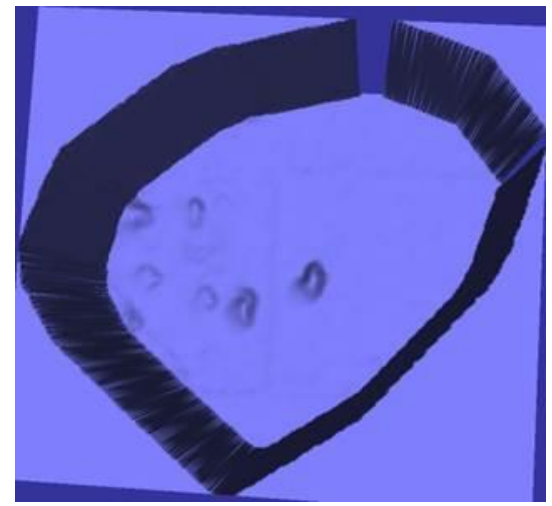

Figure 9. DEM image of the sampling area

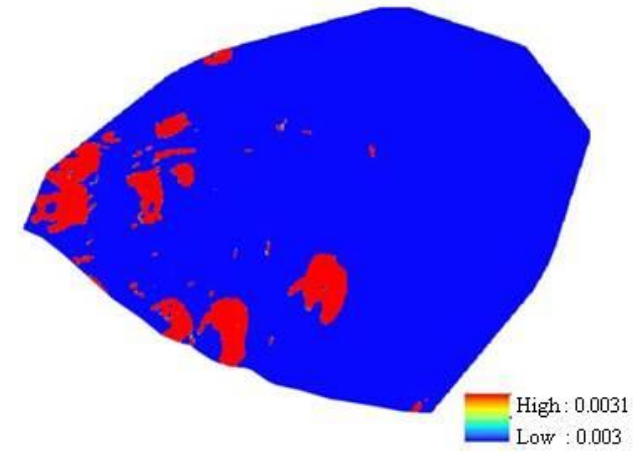

Figure 10. Feasibility image of the sampling area

\section{CONCLUSIONS}

Sampling for lunar surface exploration has become an important aspect of future deep space exploration missions. To meet the engineering requirements of automatic sampling and return for lunar exploration in China, a method for evaluating the feasibility of a lunar surface sampling area was designed in view of the unknown lunar terrain environment, uncertainty of sampling objects, and structural characteristics of the sampling manipulator and end-effector. The method took into account the topography and accessibility of the sampling area and the safety of the manipulator in the sampling process. The influence of each factor was quantified numerically, and the results were visualized to generate an admissibility image for the sampling area. The ground teleoperator can then select sampling points and control the planning of the manipulator by interpreting the admissibility image, so as to improve the efficiency of sampling area analysis. This technique will be applied to the Chinese Chang'E-5 mission.

\section{ACKNOWLEDGEMENTS}

This study was supported in part by National Natural Science Foundation of China under Grants 41771488 and 11773004.

\section{REFERENCES}

Allton J H, Bevill T J. Curatorial Statistics on Apollo Regolith Fragments Applicable to Sample Collection by Raking. Advanced Space Research, 2003, 31(11), 2305-2313.

Bugos G, Boyd J. Robotic Lunar Precursors to Apollo. AIAA Journal, 2013, 1415.

Yoseph B C, Kris Z. Drilling in extreme environments: penetration and sampling on Earth and other planets. Weinheim: Wiley-VCH Verlag GmbH \& Co. KGaA, 2009, 499-501.

Dai S W, Jia Y Z, Zhang B M, et al. Chang'E-3 scientific payloads and its checkout results (in Chinese). Sci Sin Tech, 2014, 44, 361-368. 
Yu Dengyun, Sun Jing, Ma Xingrui. Suggestion on development of Chinese space manipulator technology. Spacecraft Engineering, 2007, 16(4): 3-7 (in Chinese).

Ye Peijian, Peng Jing. Deep space exploration and its prospect in China. Engineering Science, 2006, 8(10): 13-18 (in Chinese).

Cai Ting-ni, Li Chun-lai, He Zhi-ping, et al. Experimental Ground Validation of Spectral Quality of the Chang'E-5 Lunar Mineralogical Spectrometer. Spectroscopy and Spectral Analysis, Vol 39, No 1, 257-262.

Li Wei, Sun Yuanchao, Li zongchun, et al. An improved leasesquare fitting algorithm. Science of Surveying and Mapping, Vol 42, No 1,15-19.

Denavit J, Hartenberg. A Kinematic Nation for Lower-Pair Mechanisms Based on Matrics. Journal of Applied Mechanics, 1955, 21(5), 212-215. 